\title{
Medizinische Versorgung für Menschen mit Behinderungen, die Leistungen nach dem Asylbewerberleistungsgesetzerhalten
}

Rechtliche und praktische Barrieren der Barrierefreiheit

\author{
Mirjam Schülle (M. Sc. Public Health, Universität Kassel)
}

\section{Abstract}

Der Beitrag stellt die rechtlichen und praktischen Barrieren dar, die in der gesundheitlichen Versorgung asylsuchender Menschen mit Behinderungen bestehen.

Während die Regelungen des Asylbewerberleistungsgesetzes (AsylbLG) einen medizinischen Minimalstandard normieren, soll die Aufnahmerichtlinie 2013/33/EU eine angemessene Versorgung für Menschen mit besonderen Bedürfnissen sicherstellen. Die UN-Behindertenrechtskonvention normiert zudem einen diskriminierungsfreien Zugang zu Gesundheitsleistungen. In der Praxis erweist sich vor allem die restriktive Ausgabe von Behandlungsscheinen durch die Leistungsbehörden als wesentliche Zugangsbarriere. Die alternativ eingesetzte Gesundheitskarte ermöglicht einen niedrigschwelligeren Zugang und i.d.R. wird der Leistungsumfang bedarfsgerechter ausgelegt. Die nicht flächendeckende Umsetzung durch Länder und Kommunen vergrößert allerdings die bestehende Ungleichheit in der Gesundheitsversorgung Asylsuchender.

\section{Inhalt}

1 Einführung: Barrieren der Gesundheitsversorgung für Asylsuchende mit Behinderungen ..............2

2 Gesundheitsbedarfe und -versorgung von Asylsuchenden mit Behinderungen.......................... 3

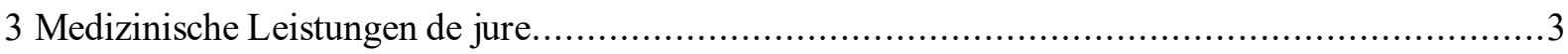

3.1 Leistungen nach dem Asylbewerberleistungsgesetz ..................................................4

3.2 Leistungen nach der Richtlinie 2013/33/EU...................................................

3.3 Leistungsrecht nach der UN-Behindertenrechtskonvention .......................................8

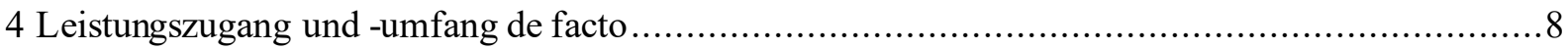

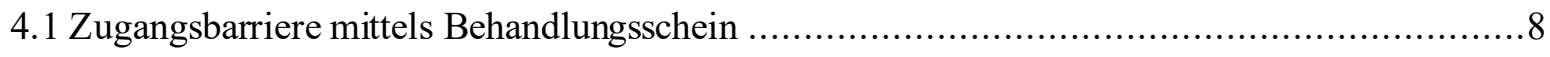

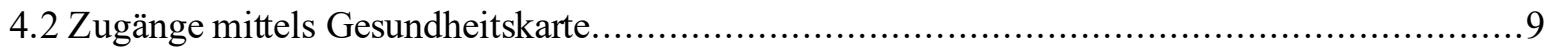

4.3 Besonderheiten für „,besonders schutzwürdige Personen“ ..................................... 10

5 Fazit: Barrierenabbau zur Sicherstellung der medizinischen Versorgung ................................ 11

\section{Schlagwörter:}

Gesundheitsversorgung, Medizinische Versorgung, Zugang zur Gesundheit, Flüchtlinge, Geflüchtete, Asylsuchende, Menschen mit Behinderungen, UN-BRK Art. 25, §§ 4, 6 Asylbewerberleistungsgesetz (AsylbLG), Aufnahmerichtlinie 2013/33/EU, Besonders schutzwürdige Personen 


\section{Einführung: Barrieren der Gesundheitsversorgung für Asylsuchende mit Behinderungen}

Der UN-Ausschuss für die Rechte von Menschen mit Behinderungen ${ }^{1}$ äußert sich in seinen abschließenden Bemerkungen über den ersten Staatenbericht Deutschlands zur Umsetzung der UN-Behindertenrechtskonvention besorgt über die bestehenden Barrieren im Zugang zur Gesundheitsversorgung, besonders für Asylsuchende und Geflüchtete mit Behinderungen. Daher empfiehlt der Fachausschuss dem Vertrag sstaat Deutschland, Pläne für die Zugänglichkeit von Gesundheitsdiensten, insbesondere für Geflüchtete, zu erstellen und umzusetzen. Geeignete Mittel seien die rechtebasierte Aus- und Fortbildung von Gesundheitsfachkräften, eine verbesserte Kommunikation und Information, die Achtung der freien, informierten Einwilligung des Einzelnen sowie die Bereitstellung von Hilfsmitteln nach universellem Design (vgl. Fachausschuss 2015). Auch in der Debatte umdas jüngst verabschiedete Bundesteilhabegesetz (BTHG) ist die Empfehlung deutlich ausgesprochen worden, den Leistungsausschluss von Geflüchteten und Asylsuchenden an Teilhabe- und Gesundheitsleistungen zu beseitigen (vgl. Schütte 2015, S. 14f.; Frehe 2016, S. 9), was allerdings keinen Eingang in das verabschiedete Gesetz gefunden hat.

Damit stellen der Fachausschuss und Wolfgang Schütte treffend Herausforderungen des Gesundheitswesens dar, auch wenn bisher die Gruppe asylsuchender Menschen mit Behinderungen kaum Beachtung gefunden hat. Aufgrund der vermutlich anhaltenden oder steigenden Zahlen geflüchteter Menschen mit besonderen gesundheitlichen Bedarfen (vgl. Razum et al. 2016b) oder Behinderungen ist eine Fokussierung auf diese Personengruppe angebracht.

Verlässliche Zahlen, wie hoch der Anteil von Menschen mit Behinderungen unterden Asylsuchenden ist, liegen nicht vor, da gegenwärtig bei der Registrierung von Asylsuchenden keine systematische Feststellung von Beeinträchtigungen stattfindet (vgl. Razum et al. 2016b; Schwalgin 2015). Laut einer Studie der Hilfsorganisationen Help Age International und Handicap International ${ }^{2}$ haben $30 \%$ der geflüchteten Menschen spezifische Bedürfnisse, jede fünfte Person ist von psychischen, sensorischen oder geistigen Einschränkungen betroffen und jede siebte Person leidet unter einer chronischen Erkrankung. Aufdieser Grundlage schätzt Handicap International, dass 10 bis $15 \%$ der geflüch teten Menschen in Deutschland eine Beeinträchtigung oder Behinderung haben (Calvotet al. 2014 nach Schwalgin 2015). Laut Dorothee Frings(2016)könnten bis zu 50 Prozent und mehr der Antragstellenden auf Asyl zu den Personen mit besonderen Bedürfnissen gehören (vgl. Frings 2016). Sehr heterogen sei dieGruppe der asylsuchenden Menschen mitBehinderungen, wie aus Berichten der Beratungsstellen hervorgeht. Sowohl Kinder und junge Erwachsene mit körperlichen, geistigen und/oder Sinnes- oder Mehrfachbehinderungen als auch Erwachsene, die Behinderungen aus Kriegsverletzungen oder nicht behandelten Verletzungen der Flucht erworben haben, sowie ältere Menschen mit Beeinträchtigungen zählen dazu (vgl. Schwalgin 2015).

Auch wenn über die Bedarfe und möglichen Defizite der gesundheitlichen Versorgung Geflüchteter empirisch zu wenig bekannt ist, können dennoch einige Aussagen aus dem Praxiswissen und den juristischen Gegebenheiten abgeleitet werden. In diesem Beitrag werden nach einem Überblick über die allgemeinen Datenlücken zur

\footnotetext{
${ }^{1}$ In diesem Beitrag wird die Definition für Menschen mit Behinderungen nach der UN-BRK zugrunde gelegt. Laut Artikel 1 UN-BRK zählen zu Menschen mit Behinderungen diejenigen, ,die langfristige körperliche, seelische, geistige oder Sinnesbeeinträchtigungen haben, welche sie in Wechselwirkung mit verschiedenen Barrieren an der vollen, wirksamen und gleichberechtigten Teilhabe an der Gesellschaft hindern können."

Aufgrund von Kritik an der offiziellen deutschen Übersetzung wurde vom NETZWERK ART IKEL 3 e.V. eine so genannte Schattenübersetzung vorgelegt. In dieser wird der Nachsatz wie folgt übersetzt; ,, die in Wechselwirkung mit verschiedenen Barrieren ihre volle und wirksame Teilhabe gleichberechtigt mit anderen an der Gesellschaft behindern können." In der englischen Originalfassung heißt es: „Persons with disabilities include those who have long-term physical, mental, intellectual or sensory impairments which in interaction with various barriers may hinder their full and effective participation in society on an equal basis with others."

${ }^{2}$ Befragt wurden 3200 syrische Geflüchtete aus Jordanien und dem Libanon (nach Schwalgin 2015).
} 
Gesundheitssituation asylsuchender Personen die rechtlichen Grundlagen der medizinischen Versorgung dargestellt und nachfolgend mit den vorliegenden Erkenntnissen über die tatsächliche Leistungsgewährung abgeglichen. Abschließend wird ein knapper Ausblick zum Abbau rechtlicher und praktischer Barrieren der Gesundheitsversorgung dieser besonders schutzwürdigen Personen gegeben.

\section{Gesundheitsbedarfe und -versorgung von Asylsuchenden mit Behinderungen}

Empirische Erkenntnisse über den Gesundheitszustand, die Gesundheitsbedarfe und die -versorgung von Asylsuchenden bestehen für Deutschland nicht. Es fehlt an einer systematischen bundesweiten Erfassung und Gesundheitsberichterstattung. Die wenigen empirischen Studien vermögen kein umfassendes Bild zu zeichnen (vgl. Razum et al. 2016a; Bozorgmehr et al. 2016a). Gleiches gilt für die Untergruppe von Menschen mit Behinderungen, die asylsuchend sind.

Aufgrund der fehlenden Daten bleiben die gesundheitlichen Auswirkungen der potentiellen Gesundheitsdeterminanten der Migrationsphasen, der sog. Prä-, Peri- und Postmigration, unerkannt. Dies gilt insbesondere für die Postmigration mit Beginn der Ankunft in Deutschland, die mit dem Asylverfahren, den Lebensumständen in Erstaufnahmeeinrichtungen oder Sammelunterkünften und einer unzureichenden Zukunftsperspektive verbunden ist. Dies sind allesamt potenziell belastende Faktoren, die sich negativ auf die Gesundheit auswirken können (vgl. Bozorgmehr et al.2016a).

Durch internationale Studien ist belegt, dass Asylsuchende und Geflüch tete eine erhöhte Krankheitslast aufweisen, besonders häufig an psychischen und chronischen Erkrankungen sowie an nicht übertragbaren Infektionskrankheiten leiden. Zwei nationale Studien (aus denen sich Kennzahlen zu nicht übertragbaren, körperlichen Krankheiten entnehmen lassen) zeigen ein Spektruman primärmedizinischen Versorgungsbedarfen: Erkrankungen der Atemwege, Schmerzen/Neuralgien, Hauterkrankungen, Zahnprobleme, Unfälle, HerzKreislauf-Erkrankungen sowie psychosomatische Beschwerden (vgl. Bozorgmehr et al. 2016a). Gesundheitliche Bedarfe liegen darüber hinaus vor allem im Bereich der psychiatrischen und psychotherapeutischen Versorgung vor(vgl. Spallek et al. 2016; Bozorgmehr et al.2016b).

Die Life-Course-Epidemiologie ${ }^{3}$ legt nahe, dass Belastungen vor und während der Flucht längerfristige sowohl physische als auch psychische gesundheitliche Auswirkungen haben können. Diese ziehen spezifische medizinische Bedarfe nach sich, die mit der Integration in die Regelversorgung und ergänzend mit zielgruppenspezifischen Angeboten gedeckt werden könnten (vgl. Razum et al. 2016a).

\section{Medizinische Leistungen de jure}

Der Zugang zu Sozial- und Gesundheitsleistungen ist für Geflüchtete grundsätzlich in Abhängigkeit ihres Aufenthaltsstatus geregelt. Anerkannte Geflüchtete, beispielsweise nach Feststellung des Bundesamtes für Migration und Flüchtlinge (BAMF) oder nach der Genfer Flüchtlingskonvention (GFK), erhalten einen Aufenthaltstitel und werden entsprechend in die sozialen Sicherungssysteme eingebunden, d.h. sie erhalten Gesundheitsleistungen analog der Gesetzlichen Krankenversicherung (GKV) (vgl. Frings 2009). Asylsuchende, die lediglich eine Aufenthaltserlaubnis oder-gestattung gewährt bekommen, sind innerhalb der ersten 15 Monate $^{4}$

\footnotetext{
${ }^{3}$ Mit der Live-Course-Epidemiologie wird versucht, Expositionen in sensiblen Lebenslagen (wie bspw. der Flucht) oder im Lebensverlauf akkumulierte kritische Ereignisse zu identifizieren, die ein späteres Auftreten von Erkrankungen begünstigen (Kreienbrock und Schach 2012).

${ }^{4}$ Bisher waren es drei Jahre, seit dem 1.3.2015 gilt die verkürzte Zeitspanne von 15 Monaten mit Inkrafttreten des Gesetzes zur Änderung des Asylbewerberleistungsgesetzes und des Sozialgerichtsgesetzes vom 10.12.2014, BGBl. 2014 I, S. 2187. Aktualisiert: Seit 01.09.2019 gilt der Zeitraum von 18 Monaten.
} 
(nach $\S 2$ AsylbLG) ihres Aufenthalts in Deutschland nicht in das System der GKV eingebunden, sondern erhalten Gesundheits- und Sozialleistungen nach dem Sondergesetz Asylbewerberleistungsgesetz (AsylbLG). Nach dem Ablaufdieser 15 Monate erhalten dieBetroffenen Leistungen in entsprechender Anwendung des zwölften Buches des Sozialgesetzbuches (SGB XII) (§ 2 I AsylbLG), d.h. Leistungen entsprechend derGKV. ${ }^{5}$

\subsection{Leistungen nach dem Asylbewerberleistungsgesetz}

Asylsuchenden, Geduldeten und Ausreisepflichtigen ${ }^{6}$ und deren Familienangehörigen wird seit der Einführung des AsylbLG 1993 eine eingeschränkte medizinische Versorgung zugesprochen. Nach § 4, Leistungen bei Krankheit, Schwangerschaft und Geburt, sind Leistungen bei akut behandlungsbedürftigen und schmerzhaften Erkrankungen mitärztlicher oder zahnärztlicher Versorgung zu gewährleisten, einschließlich der Versorgung mit Arznei- und Verbandsmitteln sowie sonstigen zur Genesung, Besserung oder Linderung von Krankheiten oder Krankheitsfolgen erforderlichen Leistungen. Eine Versorgung mitZahnersatzerfolgt nur, so weit dies im Einzelfall aus medizinischen Gründen unaufschiebbar ist. Amtlich empfohlene Schutzimpfungen und medizinisch gebotene Vorsorgeuntersuchungen werden gewährleistet, ebenso wie alle Leistungen bei Schwangerschaft und Geburt (letzteres analog zur Gesetzlichen Krankenversicherung).

Im Vergleich dazu haben Versicherte der GKV Anspruch auf Krankenbehandlung, wenn sie notwendig ist, um eine Krankheit zu erkennen, zu heilen, ihre Verschlimmerung zu verhüten oder Krankheitsbeschwerden zu linderm. Die Krankenbehandlung umfasst ärztliche Behandlung einschließlich Psychotherapie als ärztliche und psychotherapeutische Behandlung, zahnärztl iche Behandlung, Versorgung von Zahnersatz, Versorgung mit Arznei-, Verband-, Heil- und Hilfsmitteln, häusliche Krankenpflege und Haushaltshilfe, Krankenhausbehandlung, Leistungen zur medizinischen Rehabilitation und ergänzende Leistungen (vgl. § 27 SGB V).

Der $§ 4$ AsylbLG schließt dem Behandlungsanspruch vorausgehend einen Untersuchungsanspruch ein. Demnach sind die zuständigen Sozialbehörden verpflichtet, den Leistungsberechtigten eine Untersuchung durch eine Ärztin oderZahnarzt zu ermöglichen(vgl. Hohm 2015).

Strittig ist bei $\S 4$ AsylbLG die Auslegung der Worte ,akute Erkrankungen“. In der Rechtsprechung und der überwiegenden Kommentarliteratur wird eine akute Erkrankung nur in solchen Fällen ange nommen, in denen ein unvermutet auftretender, schnell und heftig verlaufender, regelwidriger Körper- oder Geisteszustand aus medizinischen Gründen der ärztlichen oder zahnärztlichen Behandlung bedarf. ${ }^{7}$ Eineakute Erkrankung steht somit im Gegensatz zu einer ,,akut nicht behandlungsbedürftigen Erkrankung“ und nicht - wie nach herrschender Meinung ${ }^{8}$ - zu einer ,, chronischen Erkrankung“, also einer sich langsamentwickelnden oder langsam verlaufenden Erkrankung. Demnach ist fraglich, ob der Begriff,,akut“einen Leistungsausschluss bei chronischen Erkrankungen bedeutet.

Wenn bei einer chronischen Erkrankung ein akuter Behandlung sbedarf oder Schmerzzustand besteht, also eine Behandlung aus medizinischen Gründen unaufschiebbar ist, sind laut der Rechtsprechung Leistungen nach $\S 4$ Abs. 1 AsylbLG zu gewähren. ${ }^{9}$ Die Einschätzung einer Erkrankung als akut behandlungsbedürftig, chronisch

\footnotetext{
5 Allerdings erhalten sie mit dieser Analogieleistung keine Eingliederungshilfen, da diese wegen ihrer finalen Zielrichtung einen nicht nur vorübergehenden Aufenthalt voraussetzen. Einige Rechtsprechungen dazu sind von Hohm 2015 zusammengestellt.

${ }^{6}$ Die detaillierte Definition der hier gemeinten und kurz zusammengefassten Personengruppen „Asylsuchende, Geduldete und Ausreisepflichtige" richtet sich nach $§ 1$ AsylbLG. Ausgeschlossen davon sind u.a. auch unbegleitete minderjährige Geflüchtete, diese stehen unter der Obhut der Jugendämter und erhalten Gesundheitsleistungen entsprechend der GKV (vgl. Turhan 2016).

${ }^{7}$ LSG NRW Urtl. v. 6.5.2013 - L 20 AY 145/11; vgl. Frerichs 2014a; Wahrendorf 2014.

${ }^{8}$ Bspw. OVG NRW Beschl. v. 7.2.2000 - 16 A 3867/99; Frerichs 2014a; Hohm 2015; Wahrendorf 2014.

${ }^{9}$ OVG NRW, Beschl. v. 20.8.2003 - 16 B 2140/02.
} 
oder akut nicht behandlungsbedürftig ist von der behandelnden Ärztin oder dem Arzt durchzuführen (vgl. Birk 2015).

Unter, ,sonstige Leistungen “nach dieser Norm sind insbesondere akut notwendige Heil- und Hilfsmittel zu zählen, wie bspw. Massagen, Krankengymnastik, Seh- und Hörhilfen sowie Prothesen (vgl. auch ff. § 6 AsylbLG). Darüber hinaus können Heil- und Genesungskuren, erforderliche Versorgungsleistungen im Rahmen eines Krankenhausaufenthaltes, Fahrtkosten und Sprachmittlerdienste zu den ,sonstigen Leistungen“ gehören (Hohm 2015).

Ein Anspruch auf Psychotherapie ist aus $\S 4$ AsylbLG nach herrschender Meinung nicht ableitbar (Ausnahme ist eine Kurzzeittherapie zur Behandlung eines akuten seelischen Schmerzzustandes ${ }^{10}$ ); auch die Hilfen zur Familienplanung oder Eingliederung shilfe für Menschen mit Behinderungen und die Hilfe zur Pflege lassen sich aus dieser Norm i.d.R. nicht schließen. Im Einzelfall ergeben sich diese Hilfen aber aus § 6 AsylbLG (vgl. Birk 2015).

„Sonstige Leistungen“"können nach § 6 AsylbLG im Einzelfall übernommen werden, wenn sie „zur Sicherung der Gesundheit erforderlich" sind. Diese Ermessensnorm ermöglicht eine weitergehende Versorgung im Krankheitsfall und umfasst ebenso Leistungen bei chronischen Erkrankungen, die überwiegend einen (tertiär)präventiven Charakter haben (vgl. Frerichs 2014b), wie ggf. auch psychotherapeutische Leistungen ${ }^{11} . \mathrm{Zu}$ sonstigen Leistungen zählen Heil-und Hilfsmittel, die nicht unter die Fälle der Akutversorgung fallen. Darunter fallen Brillen, Hörgerate, Prothesen, Rollstühle, orthopädische Schuhe und die Physiotherapie ${ }^{12}$, Sprachtherapie ${ }^{13}$ oder Ergotherapie ${ }^{14}$ genauso wie Behandlungen von Suchterkrankungen (vgl. Classen 2008). Auch Rehabilitationsleistungen sind nach dieser Norm zu übernehmen, wenn sie „zur Sicherung der Gesundheit“oder zur Deckung besonderer Bedürfnisse erforderlich sind ${ }^{15}$, ebenso Maßnahmen zur Eingliederungshilfe, bspw. Leistungen zur Teilhabe am Leben in der Gemeinschaft (vgl. Hohm 2015) ${ }^{16}$ und Leistungen bei Pflegebedürftigkeit, wenn sie „unabweisbar erforderlich“ sind. Leistung en der Frühförderung und sonstige Hilfen für Kinder mit Behinderungen ${ }^{17}$ wurden vom Verwaltungsgericht Sigmaringen zugesprochen ${ }^{18}$. Zudem besteht grundsätzlich die Verpflichtung zur Übernahme der Sprachmittler- und Dolmetscherkosten, wenn ansonsten der Anspruch auf Krankenhilfe nichterfüllt werden $\mathrm{kann}^{19}$. Überwiegend wird in der Kommentarliteratur die Meinung vertreten, dass $\S 6$ Abs. 1 restriktiv und als Ausnahmevorschrift auszulegen ist. Bei der medizinischen Leistungsgewährung sind die $\S \S 4$ und 6 zusammen zu denken (vgl. Herbst 2015), was aber von der Rechtsprechung teilweise verkannt wird (vgl. Müller-Krah 2012).

Das AsylbLG enthält - anders als die gesetzlicheKrankenversicherung - keine Rechtsgrundl age fürZuzahlungen und Eigenleistungen ${ }^{20}$. Entsprechend sind von Krankenhäusern, Apotheken, Krankentransporten keine Zuzahlungen u.Ä. zu fordern und die Leistungen sind nach §§ 4, 6 vollständig mit dem Sozialamt abzurechnen. Beispielsweise ist dies u.a. für Brillen, Hörgeräte, Physiotherapie, orthopädische Schuhe, Zahnersatz, Fahrten zur

\footnotetext{
${ }^{10}$ OVG Niedersachsen, Beschl. v. 22.9.1999 - 4 M 3551/99.

${ }^{11}$ OVG Lüneburg, Urt. v. 22.9.99, 4 M 3551/99.

${ }^{12}$ SG Gießen, Urt. v. 10.08.06 S18 AY 6/06.

${ }^{13}$ VG Augsburg, Beschl. v. 12.2.2004 - Au 9 K 03.1215.

${ }^{14}$ Ebd.

${ }^{15}$ OVG Saarland, Urt. v. 24.4.2006 - 3 W 3/06.

16 VG Sigmaringen, Urt. v. 2.4.2003-5 K 781/02.

${ }^{17}$ Vertiefend zu Kindern mit Behinderungen siehe Kenan Engin in diesem Band.

${ }^{18}$ Ebd.

${ }^{19}$ VG Saarland, Urt. V. 29.12.2000 - 4 K 66/99.

${ }^{20}$ Mit den Geldleistungen des AsylbLG sind Zuzahlungen nicht zumutbar bzw. fallen die Leistungsberechtigten sonst unterhalb des Existenzminimums.
} 
ärztlichen Krankenbehandlung ${ }^{21}$, Sprachmittler-, und Dolmetscherkosten und rezeptfreie Medikamente zu berücksichtigen(vgl. Classen 2016).

Diese medizinische Grundversorgung auf niedrigem Niveau nach AsylbLG soll sich - nach dem Willen des Gesetzgebers $^{22}$ - nicht an den Maßstäben des Sozialhilferechts (SGB XII) bzw. der gesetzlichen Krankenversicherung (SGB V) orientieren. Die dahinterliegende Begründung des Gesetzgebers von 1993 ist, dass die Versorgung nach AsylbLG nur Menschen betrifft, die sich voraussichtlich vorübergehend in Deutschland aufhalten. Das Landessozialgericht Baden-Württemberg ${ }^{23}$ hat den Leistungsumfang demnach folgendermaßen interpretiert: „Hilfeempfängernach dem AsylbLG haben im Rahmen der Leistungen bei Krankheitnach $\S 4$ Abs. 1 AsylbLG keinen Anspruch auf optimale und bestmögliche Versorgung ${ }^{24}$, sondern nur auf Hilfe bei akuten Erkrankungen und Schmerzzuständen. Langwierige Behandlungen, die wegen der absehbar kurzen Dauer des weiteren Aufenthalts vorrausichtlich nicht abgeschlossen werden können, begründen keine Leistungsverpflichtung. Über die Auffang- und Öffnungsklausel des $\S 6$ Abs. 1 AsylbLG können nur unerlässliche, d.h. unverzichtbare Leistungen [Herv. v. Verf.] erbracht werden. Dies gilt auch im Falle von chronischen Erkrankungen.“ Dieser Leitsatz enthält, in programmatischer Kürze zusammengefasst, die Leitlinie vieler Gerichte und Behörden für die Gewährung von Gesundheitsleistungen für Geflüchtete. Eberhard Eichenhofer (2013) stellt zu Recht die Frage, woraus die Gerichte diese Gewissheit angesichts eines noch schwebenden und daraus auch erfolgreich endenden Asylverfahrens beziehen und mit welchem Recht hier ein negativer Ausgang der Hauptsache als gegeben unterstellt wird (vgl. Eichenhofer 2013).

Die Vereinbarkeit des abgesenkten Leistungsanspruchs auf Gesundheitsleistungen nach § 4 AsylbLG mit höherrangigem Recht ist juristisch umstritten (stellv. vgl. Kaltenborn 2015, Rixen 2015, Frerichs 2014a, Eichenhofer 2013). Verfassungsrechtliche Bedenken ergeben sich aus dem Sozial staatsprinzip (i.V.m. Art. 2 Abs. $1 \mathrm{GG}$ ) und der Pflicht des Staates zum Schutz der Gesundheitund körperlichen Unversehrtheit (Art. 2 Abs. 2 Satz 1 GG). Spätestens nach der Grund satzentscheidung des Bundesverfassungsgerichts 2012 zum Leistungsrecht auf Gewährung eines menschenwürdigen Existenzminimums (Art. 1 Abs. 1 GG i.V.m. Art. 20 Abs. 1 GG) erhielt die Frage der gesundheitlichen Versorgung eine leistungsrechtliche Dimension (vgl. Eichenhofer 2013). Nach der Rechtsprechung des BVerfG ist die Voraussetzung für die unterschiedliche Sicherstellung der existenznotwendigen Leistungen eine signifikante Feststellung (in einem transparenten und nachvollziehbaren Verfahren) abweichender Bedarfe. Einen herabgesetzten medizinischen Bedarf dieser vulnerablen Personengruppe festzustellen, dürfte im Ergebnis nicht gelingen (so auch Schütte2015, S. 14), besonders da erste empirische Erkenntnisse auf einen erhöhten Bedarfhinweisen.

Zusammengefasst ist zu konstatieren, dass Rechtsprechung und Rechtskommentierungen unterschiedliche Interpretationen der medizinischen Versorgung nach AsylbLG haben. Zum einen wird von einer sehr einschränkten Leistungsgewährung ausgegangen (wie das zitierte Beispiel des LSG BaWü) und zum anderen von einem Leistung sumfang annähernd nach SGB V (vgl. Welti in diesem Band). Letzteres insbesondere dann, wenn die Auslegung der Normen unter Berücksichtigung des Verfassungs-, Europa- sowie Völkerrechts erfolgt.

\footnotetext{
${ }^{21}$ LSG Sachsen-Anhalt, Beschl. v. 9.3.2015 - L 8 SO 23/14 B ER.

22 BT-Drs. 12/5008; BT-Drs. 12/4451.

${ }^{23}$ LSG BaWü, Urt. v. 11.1.2007 - L 7AY 6025/06.

${ }^{24}$ Frerichs gibt kritisch zu bedenken, diese Formulierung sei vor dem Hintergrund der medizinischen Notversorgung nach $\$ 4$ und den Grundsätzen der GKV (vgl. § 12 SGB V) eine „Leerformel ohne Gehalt“ (vgl. Frerichs 2014b, Rn. 70).
} 


\subsection{Leistungen nach der Richtlinie 2013/33/EU}

Für die Gesundheitsversorgung von asylsuchenden Menschen während der Dauer ihres Asyl- bzw. Anerkennungsverfahrens - d.h. nicht für geduldete Menschen ${ }^{25}$ - gilt über das AsylbLG hinaus die Aufnahmerichtlinie 2013/33/EU (EU-RL) ${ }^{26}$. Danach könnten medizinische Leistungen auf einen Basisschutz reduziert werden, allerdings gilt dies nicht für Menschen mit besonderen Bedürfnissen, sog. vulnerable Personengruppen.

Die EU-RL verpflichtet die Mitgliedsstaaten, die „spezielle Situation von schutzbedürftigen Personen wie Minderjährigen, unbegleiteten Minderjährigen, Behinderten ${ }^{27}$ [Herv. v. Verf.], älteren Menschen, Schwangeren, Alleinerziehenden mit minderjährigen Kindern, Opfern des Menschenhandels, Personen mit schweren körperlichen Erkrankungen, Personen mit psychischen Störungen und Personen, die Folter, Vergewaltigung oder sonstige schwere Formen psychischer, physischer oder sexueller Gewalt erlitten haben, wie z.B. „Opfer der Verstümmelung weiblicher Genitalien“(Art. 21), zu berücksichtigen. Diesen schutzbedürftigen Personen sind gemäß EU-RL die „erforderliche medizinische Versorgung [Herv. v. Verf.] oder sonstige Hilfen, einschließlich erforderlichenfalls einer geeigneten psychologischen Betreuung“ (Art. 19 Abs. 2), zu gewähren. Der Begriff „erforderliche“ Leistung en dürfte sich mit den „notwendigen“ Leistungen im Sinne der GKV decken, woraus ein Leistungsanspruch entsprechend der GKV (§ 27 SGB V) abzuleiten ist (vgl. Frings 2009, 2016). Nach einer solchen richtlinienkonformen Auslegung steht asylsuchenden Menschen mit Behinderungen ein Leistungsniveau der medizinischen und rehabilitativen Versorgung mindestens nach § 6 AsylbLG zu (vgl. Frerichs 2014b). Der Ermessensspielraum des $\S 6$ AsylbLG reduziert sich somit aufgrund der EU-RL für besonders schutzbedürftige Personen auf Null ${ }^{28}$ Die Behörden sind damit zur Leistungsgewährung nach $\S 6$ AsylbLG verpflichtet, wenn die Entscheidung rechtsfehlerfrei sein soll.

Die Aufnahmerichtlinie gilt für alle Personen, die einen Antrag auf internationalen Schutz gestellt haben, auch wenn über diesen noch nicht entschieden wurde. Bis zum 20. Juli 2015 (Art. 31) hatten die Mitgliedsstaaten die Verpflichtung, die Richtlinie vollständig in nationales Recht umzusetzen. Die Bundesrepublik Deutschland(BRD) hat dies bisher nicht verwirklicht, die Europäische Kommission hat daher im September 2015 ein Vertragsverletzungsverfahren gegen Deutschland eingeleitet (Europäische Kommission, Pressemitteilung vom 23.9.2015). Die einzelnen Bestimmungen der Richtlinie können unmittelbar anwendbar sein. Die bestimmten Voraussetzungen ${ }^{29}$ dafür, sollten bei der Gesundheitsversorgung gegeben sein.

Auf die Einhaltung der sozialen Mindeststandards nach der EU-RL können sich allerdings nur Personen berufen, die nach einer Einzelfallprüfung ihrer Situation als besonders hilfebedürftig anerkannt sind. Bisher existiert ein solches einfachgesetzliches Anerkennungsverfahren nicht.

\footnotetext{
${ }^{25}$ Ein erhöhter Leistungsanspruch als § 4 AsylbLG sollte sich für geduldete Menschen nach der UN-BRK ergeben (vgl. folgendes Kapitel 3.3).

${ }^{26}$ Vorgänger Richtlinie 2003/9/EG, welche ebenso bisher von der BRD nicht umgesetzt wurde.

${ }^{27}$ Eine Definition der Personengruppe „Behinderte“ ist in der EU-RL nicht aufgeführt. Allerdings sind die Vertragsstaaten zur Identifizierung der Personengruppe verpflichtet, d.h. spätestens bei diesem Verfahren ist eine Definition zugrunde zu legen (siehe 4.3). Um eine einheit liche Definition der Personengruppe Menschen mit Behinderungen innerhalb der Mitgliedsstaaten der EU zu erreichen, ist die Definition der UN-BRK zielführend.

${ }^{28}$ BT-Drs. 18/7831, S. 5, BT -Drs. 18/9009, S. 3.

${ }^{29}$ Voraussetzungen: 1. Die Umsetzungsfrist ist ohne vollständige Umsetzung verstrichen, 2. Die Richtlinienbestimmungen sind inhaltlich hinreichend bestimmt und unbedingt (vgl. u.a. EuGH, Urt. v. 19.1.1982 - 8/81; EuGH, Urt. v. 24.11.2011 - C468/10), 3. Die Bestimmungen verleihen dem Einzelnen Rechte gegenüber dem Mitgliedsstaat (vertiefend siehe Hainthaler 2015).
} 


\subsection{Leistungsrecht nach der UN-Behindertenrechtskonvention}

Die UN-Behindertenrechtskonvention (UN-BRK) ist ein 2009 von Deutschland ratifizierter völkerrechtlicher Vertrag, der die herkömmlichen Menschenrechte für die Lebenssituation von Menschen mit Behinderungen konkretisiert und aus ihrer Perspektive regelt. Sie ist fürjeden Menschen in Deutschland anzuwenden. Das soziale Menschenrecht aufGesundheit findetsich in der UN-BRK in Art. 25 wieder: ,States Parties recognize that persons with disabilities have the right to the enjoyment of the highest attainable standard of health withoutdiscrimination [Herv. v. Verf.] on the basis of disability. States Parties shall take all appropriate measures to ensure access for persons with disabilities to health services [Herv.v. Verf.] that are gender-sensitive, including health-related rehabilitation. ${ }^{\text {30 }}$

Demnach ist jede Diskriminierung aufgrund einer Behinderung im Bereich der Gesundheitsfürsorge unzulässig (vgl. Lachwitz 2013). Die eingeschränkten Behandlungsansprüche nach dem AsylbLG zielen nicht auf eine Benachteiligung aufgrund der Behinderung sondern aufgrund des Aufenthaltsstatus ab. Frings ist der Meinung, dass aufgrund des Zusammentreffens der Behinderung und des aufenthaltsrechtlichen Status diese Menschen dennoch mittelbar diskriminiert werden, insbesondere im Zugang zur gleichberechtigten und bedarfsgerechten medizinischen Versorgung (vgl. Frings 2009). Für die Rechtsanwendung und Rechtsprechung sollte $\S 25$ UNBRK zumindest Berücksichtigung finden ${ }^{31}$ wodurch ein Mindeststandard demnach nicht unterschritten werden darf, was bei § 4 AsylbLG sehr frag würdig ist. Grundsätzlich widersprechen die Leistungsein schränkungen nach dem aufenthaltsrechtlichen Status Art. 1 UN-BRK, der den vollen und gleichberechtigten Anspruch aller Menschenrechte und Grundfreiheiten füralle Menschen mit Behinderungen zubilligt.

\section{Leistungszugang und -umfang de facto}

Direkt nach der Ankunft in Deutschland sind asylsuchende Menschen in Erstaufnahme- oder Nebeneinrichtungen untergebracht und sollen von den Einrichtungen medizinisch versorgt werden, für deren Durchführung das jeweilige Bundesland verantwortlich ist (vgl. Wächter-Raquet 2016a). Nach der erfolgten Zuweisung an eine Kommune ist diese für die medizinische Versorgung verantwortlich. Der gesundheitliche Sicherheitsauftrag ist sowohl auf kommunaler als auch auf Länderebene unterschiedlich ausgestaltet ${ }^{32}$ die Leistungsberechtigten erhalten Zugang zum Gesundheitssystem entweder mit dem so genannten Behandlung sschein oder über eine Gesundheitskarte (GK). ${ }^{33}$

\subsection{Zugangsbarriere Behandlungsschein}

Die Inanspruchnahme von Gesundheitsleistungen erfolgt vielerorts über einen persönlichen Antrag an die zuständige Sozialbehörde und die anschließende Ausgabe eines Behandlungsscheines (auch Krankenschein genannt $)^{34}$. Bei einer Folgebehandlung oder Facharztüberweisung muss ein Behandlungsschein erneut beantragt werden. Manche Sozialbehörden haben dieses Verfahren vereinfacht und stellen pauschal einen Schein pro Quartal

\footnotetext{
${ }^{30}$ Offizielle deutsche Übersetzung: „Die Vertragsstaaten anerkennen das Recht von Menschen mit Behinderungen auf das erreichbare Höchstmaß an Gesundheit ohne Diskriminierung aufgrund von Behinderung [zu genießen laut Schattenübersetzung]. Die Vertragsstaaten treffen alle geeigneten Maßnahmen, um zu gewährleisten, dass Menschen mit Behinderungen Zugang zu geschlechtsspezifischen [gender-sensiblen laut Schattenübersetzung] Gesundheitsdiensten, einschließlich gesundheitlicher Rehabilitation, haben." Artikel 25 UN-BRK in der offiziellen deutschen Übersetzung.

${ }^{31} \mathrm{Zu}$ der Bedeutung der UN-BRK für die Leistungsgewährung Asylsuchender gibt u.a. das BSG-Urteil vom 29. April 2010 B 9 SB 2/09 R Aufschluss. Siehe auch Gagel: Umsetzung der UN-Behindertenkonvention - hier: Recht auf Feststellung des GdB und des Schwerbehindertenstatus für lediglich geduldete Ausländer; Beitrag A 13/2010 unter www.reha-recht.de; 21.12.2010.

${ }^{32}$ Zur Durchführung des AsylbLG gelten uneinheitliche Verwaltungsvorschriften der Länder, die den Leistungsbehörden die Auslegung und den Leistungsumfang des $§ 4$ vorgeben.

${ }^{33}$ In der Literatur auch elektronische Gesundheitskarte (eGK) genannt.

${ }^{34}$ Der Behandlungsschein ist ein Relikt des Krankenversicherungssystems vor 1995, bis die Karte als Abrechnungsinstrument eingeführt wurde.
} 
aus. Mit einem Behandlungsschein können die Betroffenen eine Ärztin oder einen Arzt aufsuchen. Die Kostenabrechnung verläuft über die zuständige Sozialbehörde. Im medizinischen Notfall kann eine Behandlung ambulant oder stationär ohne Vorlage eines Behandlungsscheineserfolgen und die Abrechnung erfolgt auch dann unmittelbar gegenüber dem Kostenträger, der Sozialbehörde. Kritisiert wird am Behandlungs-scheinverfahren, dass medizinische Laien (Sozialamtsmitarbeitende) über die Ausstellung entscheiden, ohne notwendige medizinische Kenntnisse zu haben. Ein solches Entscheidungsverfahren über den Untersuchungsanspruch ist gemäß § 4 AsylbLG (vgl. Kapitel 3.1) rechtlich unzulässig, dennoch ist dies in der Behördenpraxis vielerorts üblich. Der Zugang mittels Behandlungsschein erfordert für die Betroffenen zudem einen irregulären Aufwand, sodass von einer strukturellen Zugangsbarriere gesprochen wird (vgl. Bozorgmehr und Razum 2015).

Aus der Praxis der Unterstützungseinrichtungen für Asylsuchende ist bekannt, dass vielfach offensichtlich notwendige Behandlungsscheine nicht genehmigt werden. Die praktische Durchsetzung therapeutischer Behandlungen verlangt von den Betroffenen oftmals gesteigerte Anstrengungen, langwierige amtsärztliche Gutachterverfahren und die Inanspruchnahme behördlichen und gerichtlichen Rechtsschutzes. Empirisches Wissen über die Voraussetzungen und Kriterien, nach denen die Behandlungsscheinvergabe erfolgt, besteht nicht. Dieses Wissen wäre besonders im Fall der Vergabe der Behandlungsscheine gemäß der Öffnungsklausel in $\S 6$ AsylbLG relevant, wodurch verbesserte Aussagen zum Leistungszugang und -umfang u.a. zu Heil- und Hilfsmitteln sowie zu Teilhabeleistungen für Menschen mit Behinderung, die Leistungen nach dem AsylbLG beziehen, getroffen werden könnten.

Der Umstand, dass asylsuchende Menschen mit Behinderungen Krankenscheine anstatt Gesundheitskarten erhalten - letzteres wie alle anderen Bewohnerinnen und Bewohner der BRD-, kann als eine strukturelleBarriere im Zugang zu Gesundheitsleistungen und als unzulässige Diskriminierung gewertet werden.

\subsection{Zugänge mittels Gesundheitskarte}

Einige Länder und Kommunen haben den Zugang zum Gesund heitssystem für Asylsuchende analog zu gesetzlich Versicherten regulär mittels einer GK organisiert. Seit 2005 erhalten in Bremen alle Asylsuchenden aufder Basis von $\S 264$ SGB V eine GK der AOK Bremen/Bremerhaven. Die Ausgabe der GK ist Teil eines Gesamtkonzeptes zur Gesundheitsversorgung Geflüchteter, welches als Bremer Modell bekannt ist ${ }^{35}$. Ziel des Bremer Modells ist, eine vereinfachte und verbesserte Gesundheitsversorgung für alle Geflüchteten, welche diskriminierung sfrei und zeitnah weitestgehend in die Regelversorgung integriert werden. Mit der GK haben die Betroffenen einen niedrigschwelligen und selbstbestimmten Zugang zu allen Einrichtungen des Gesundheitswesens und sind diesbezüglich sozialversicherten Menschen gleichgestellt. Grundlage des Leistungsumfangs nach dem Bremer Modell sind die gesetzlich vorgegebenen $\S \S 4,6$ AsylbLG; diese werden allerdings dahingehend sachgerecht interpretiert, dass eine Versorgung annähernd entsprechend dem Leistungskatalog der gesetzlichen Krankenversicherung erfolgt. Ausgeschlossen sind strukturierte Behandlungen bei chronischen Erkrankungen (Disease-Management-Programme), künstliche Befruchtung, Akupunkturbehandlung, Entbindungsgeld, Mutterschaftsgeld und die Kostenübernahme von Behandlungen im Ausland. Im Rahmen eines Genehmigungsverfahrens über das Gesundheitsamt (mit Gutachten) sind erweitert Psychotherapie (nur Kurzzeittherapie), Zahnersatz, kieferorthopädische Behandlungen und besonders für Menschen mit Behinderungen relevante Leistungen wie Vorsorgekuren, Rehabilitationsmaßnahmen, Sehhilfen und Hilfsmittel möglich (vgl. Mohammadzadeh et al. 2016). Diesem Vorbild folgte im Jahr 2012 die Freie und Hansestadt Hamburg.

\footnotetext{
${ }^{35}$ Dieses Gesundheitskonzept entstand bereits 1993 und wird seit dem kontinuierlich angewendet und ggf. erweitert. Vertiefend zum Bremer Modell siehe Mohammadzadeh 1993; Lelgemann und Mohammadzadeh 2015; Mohammadzadeh et al. 2016 .
} 
Seit der Verabschiedung des Asylverfahrensbeschleunigungsgesetzes im Oktober 2015 wurde den Ländern durch die Änderungen des § 264 Abs. 1 SGB V die Einführung der GK erleichtert. Die Länder können demnach Rahmenverträge für ihre Kommunen aushandeln und Krankenkassen verpflichten, die Verträge mit den Kommunen abzuschließen. Dies ist insbesondere für Flächenländer relevant. Seitdem ${ }^{36}$ haben NordrheinWestfalen (NRW), Schleswig-Holstein, Berlin, Brandenburg, Niedersachsen, Rheinland-Pfalz und Thüringen Rahmenverträge abgeschlossen. Die Kommunen treten diesen Verträgen allerdings nur zögerlich bei, in NRW sind es 20 von 400 Kommunen, in Niedersachsen ledigl ich eine Kommune. Derzeit ist ein sog. Flickenteppich an unterschiedlichen Leistungszugängen zu konstatieren. Einige Kommunen arbeiten nach wie vor mit Behandlungsscheinen, andere mit GK; ebenso divers ist es in den Ländern gestaltet. Gleichermaßen unterscheiden sich die Inhalte der Rahmenverträge, was insbesondere die Interpretation der $\S$ 4, 6 AsylbLGbetrifft, wie auch die Finanzierungsteilung und Durchsetzung des Landes gegenüber den Kommunen. Als einziges Land hat bspw. Schleswig-Holstein alle Kommunen per Weisung zum Beitritt verpflichtet, allerdings trägt das Land auch $90 \%$ der Kosten. Thüringen hat Ende 2016 einen Rahmenvertrag abgeschlossen mit einem sach-, und bedarfsgerechten Leistungskatalog. Seit Anfang 2017 werden in Thüringen die GK ausgegeben, das Land trägt die Kosten, die Kommunen sind zum Beitritt verp flichtet. (vgl. Wächter-Raquet 2016 $\mathrm{a}^{37}$ ).

Derzeit ist die Kostenfrage bei der Einführung der GK die strittigste. Kommunen befürchten durch die Verwaltungskosten der Krankenkassen erhöhte Ausgaben. Empirisch belegt sind bisher lediglich Kosteneinsparungen durch die Einführung der GK (vgl. Bozorgmehrund Razum 2015).

Vorteil der GK ist ein bedarfsgerechter, niedrigschwelliger und gleichberechtigter Zugang zu allen erforderlichen Gesundheitsleistungen für die Betroffenen und zudem ein vereinheitlichter Leistungsumfang. Darüber hinaus verringert die GK den Entscheidungs- und Prüfaufwand bei den Sozialbehörden und normalisiert den Abrechnungsaufwand bei den Leistungserbringenden.

Durch die vermehrte aber unterschiedliche Einführung der GK 2015/16 ist allerdings zu konstatieren, dass die bestehende Ungleichheit innerhalb der Gesundheitsversorgung Geflüchteter zugenommen hat. Um der gesundheitlichen Ungleichheit für Geflüchtete durch unterschiedlichen und verringerten Leistungszugang entgegenzuwirken, ist eine bundeseinheitliche Regelung geboten. Eine solche Regelung würde insbesondere auch flächendeckend Barrieren für Menschen mit Behinderungen beim Zugang zum Gesundheitssystem abbauen und einen bedarfsgerechten Leistungsumfang für diese besonders schutzwürdige Personengruppe gewährleisten.

In den Kommunen und Ländern, die den Leistungszugang über die GK organisiert haben, haben asylsuchende Personen mit Behinderungen einen verbesserten und weitestgehend UN-BRK-konformen Zugang zu medizinischen Leistungen. Entsprechend ist für diese Personengruppe in einigen dieser Kommunen und Ländem auch der Leistungsumfang sachgerecht, nämlich annähernd nach GKV-Leistungen, ausgestaltet.

\subsection{Besonderheiten für „,besonders schutzwürdige Personen“}

Art. 21 und 22 der EU-Richtlinie 2013/33 verpflichten zur Einrichtung eines speziellen Verfahrens zur Identifizierung von Personen mit besonderen Bedürfnissen. Das BAMF weist darauf hin, dass ein solches Prüfungsverfahren, in der Praxis auch Clearingverfahren genannt, bei jeder Antragstellung (also in den Erstaufnahmestellen der Länder) einzuhalten ist (vgl. BMAF 2015, S. 2).

Die Erfahrungswerte aus der Praxis zeigen jedoch, dass bisher ein solches Clearingverfahren lediglich bei so genannten Unbegleiteten Minderjährigen Flüchtlingen (UMF) fest etabliert ist, bei allen weiteren besonders schutzbedürftigen Personen allerdings nicht. Für Menschen mit Behinderungen sind einige wenige Modellprojekten bekannt, die ein solches Prüfverfahren zu etablieren versuchen. In einer Clearingstelle in Hamburg erhalten besonders schutzbedürftige Personen eine Bescheinigung über dieentsprechende Anerkennung.

\footnotetext{
${ }^{36}$ Stand Februar 2017

${ }^{37}$ Eine grundlegende Übersicht zu dem Umsetzungsstand der GK in den einzelnen Ländern bieten Wächter-Raquet (2016a, 2016b) und in aktualisierter Fassung die Plattform www.gesundheit-gefluechtete.info.
} 
Die Bescheinigung dient dem erleichterten Zugang zu Sozialleistungen (was insbesondere in Kommunen und Ländern mit Behandlungsscheinvergabe relevant ist); die Praxis weist allerdings Mängel bei der Umsetzung auf (vgl. Niethammer und Aumann 2014). In Berlin wird seit 2009 ein dreistufiges kooperatives Verfahren entwickelt und versucht, dieses umzusetzen. Im ersten Schritt erfolgen die Ermittlung von Schutzbedürftigkeitsmerkmalen und die Weiterleitung an die entsprechende Fachstelle. Im zweiten Schritt wird in der Fachstelle eine Gesundheitsprüfung und Erstdiagnostik durchgeführt und auf dieser Grundlage eine Bescheinigung über die Zugehörigkeit zu einer der Gruppen der Schutzbedürftigen ausgestellt. Im dritten Schritt soll diese Bescheinigung bei den Sozialbehörden zur richtlinienkonformen Gewährung gesundheitlichen und materiellen Leistungen führen. Ähnlich wie in Hamburg istder dritte Verfahrensschritt im Ergebnis nicht gewährleistet und die Leistungen werden von den Leistungsträgern nur bedingt und verzögert erbracht (vgl. ebd.).

Zusammenfassend ist aus der Praxis bekannt, dass die Leistungsgewährung unter Berücksichtigung der besonderen Schutzbedürftigkeit mittels Behandlungsscheinverg abe grundsätzlich unzureichend erfüllt wird, selbst in Modellprojekten mit Bescheinigungen dieser besonderen Schutzbedürftigkeit. Mutmaßlich kann dies an mangelndem Wissen und fehlenden Weisungen zur richtlinienkonformen Umsetzung innerhalb der Sozialbehördenliegen.

\section{Fazit: Barrierenabbau zur Sicherstellung der medizinischen Versorgung}

Wie aus den vorangegangenen Kapiteln ersichtlich ist, bestehen sowohl rechtlich als auch praktisch unterschiedliche und vielseitige Barrieren ${ }^{38}$ bei der Sicherstellung der medizinischen Versorgung asylsuchender Menschen, insbesondere auch für Menschen mit Behinderungen.

Den rechtlich zugesicherten Schutz bei Krankheit nach dem AsylbLG fasst Eichenhofer als „, fragmentarisch, punktuell, vorläufig und rechtlich ungesichert" zusammen (Eichenhofer 2013, S. 17), er wird als eine bedeutende Barriere einer bedarfsgerechten Gesundheitsversorgung verstanden.

Die rechtlich zugesicherten erweiterten Gesundheitsleistungen nach GKV-Niveau für besonders schutzwürdige Personen und Menschen mit Behinderungen, wie sie sich aus Art. 19 EU-RL 2013/33 und Art. 25 UN-BRK ergeben, haben in der behördlichen und richterlichen Praxis bisher keine Geltung erfahren. Wenn § 6 AsylbLG sachgemäß ausgelegt und genehmigt wird, kann gesagt werden, dass die EU-RL weitestgehend praktisch umgesetzt wird, rechtlich allerdings nicht sauber verankert ist. Eine Ermessensleistung, wie sie § 6 AsylbLG normiert, ist laut EU-RL nicht vorgesehen.

Angesichts der defizitären Ausgestaltung der $\S \S 4,6$, AsylbLG ist der Gesetzgeber angehalten, die strukturelle Unterversorgung für Asylsuchende, insbesondere der besonders schutzbedürftigen Personengruppen, - unter der Berücksichtigung des höherrangigen Rechts - grundlegend neu zu regeln (so auch Birk 2015; Frerichs 2014a; Eichenhofer 2013). ${ }^{39}$ Somit würden Mehrfachdiskriminierungen im Zugang zur umfassenden Gesundheitsversorgung für Menschen mit Behinderungen, die Leistungen nach dem AsylbLG beziehen, de jure abgebaut werden. In diesem Zug könnte die fragliche föderale Regelung der Gesundheitsversorgung Asylsuchender mit einer bundeseinheitlichen Ausgestaltung - wie es in der Gesundheitspolitik grundsätzlich gehandhabt wird - ersetzt werden.

\footnotetext{
${ }^{38}$ Für die USA wurden in einer ersten Übersichtsarbeit weitreichende Barrieren im Zugang zum Gesundheitssystem für Geflüchtete empirisch bestätigt. Aus Sicht der Betroffenen sind demnach drei Hauptbarrieren identifiziert worden: 1. der unzureichende staatlich gewährte Versicherungsschutz, 2. Sprachbarrieren mit den Akteurinnen und Akteuren des Gesundheitswesens und 3. das komplexe Gesundheitssystem als solches (Mirza et al. 2014). Laut den existierenden Hinweisen könnte dies durchaus für Deutschland übertragbar sein, eine empirische Überprüfung dessen steht aber noch aus. ${ }^{39}$ Bisher sind die Oppositionsparteien mit wiederholten Anträgen (BT -Drs. v. 7.10.2014,18/2736, BT -Drs. v. 28.1.2016, 18/7413) mit einem solchen Vorhaben gescheitert.
} 
Bis dahin sind die Leistungsbehörden und Gerichte zur EU-RL- und UN-BRK-konformen Auslegung der einschlägigen nationalen Normen verpflichtet (vgl. auch Turhan 2016), wie die Bundesregierung im März und Juni 2016 bestätigte $^{40}$ (vgl. Voigt 2016). Für asylsuchende Menschen mit Behinderungen (und alle besonders schutzwürdigen Personengruppen) ist somit eine Leistungsgewährung nach GKV-Niveau geboten. Dies ist insbesondere dann zu beachten, wenn der Leistungszugang mit den herkömmlichen Behandlungsscheinen organisiert ist. In den Ländern und Kommunen, die eine GK ausgeben, ist der Leistungszugang diskriminierungsfrei gestaltet und der Leistungsumfang entspricht vielerorts annähernd dem GKV-Niveau. In diesem Fall wird den Anforderungen aus der UN-BRK und EU-RL weitgehend entsprochen.

\section{Literatur}

Birk, Ulrich-Arthur. 2015. § 4 AsylbLG. In Sozialgesetzbuch XII. Sozialhilfe; Lehr-und Praxiskommentar, Hrsg. RenateBieritz-Harder, Wolfgang Conradis, und Stephan Thie. Baden-Baden: Nomos.

Bozorgmehr, Kayvan und Oliver Razum. 2015. Effect of Restricting Access to Health Care on Health Expenditures among Asylum-Seekers and Refugees: A Quasi-Experimental Study in Germany, 1994-2013. PloS one 10 (7): e0131483. doi: 10.1371/journal.pone.0131483.

Bozorgmehr, Kayvan, Amir Mohsenpour, Daniel Saure, Christian Stock, Adrian Loerbroks, Stefanie Joos, und Christine Schneider. 2016a. Systematische Übersichtund "Mapping" empirischer Studien des

Gesundheitszustands und der medizinischen Versorgung von Flüchtling en und Asylsuchenden in Deutschland (1990-2014). Bundesgesundheitsblatt, Gesundheitsforschung, Gesundheitsschutz 59(5): 599-620. doi: 10.1007/s00103-016-2336-5.

Bozorgmehr, Kayvan, Stefan Nöst, Heidrun M. Thaiss, und Oliver Razum. 2016b. Die gesundheitliche Versorgungssituation von Asylsuchenden. Bundesweite Bestandsaufnahme über die Gesundheitsämter. Leitthema: Gesundheit und Versorgung von Asylsuchenden und Geflüchteten 59 (5): 545-555.

Bundesamt für Migration und Flüchtlinge. 2015. Leitfaden zur unmittelbaren innerstaatlichen Anwendung der Richtlinie 2013/32/EU des Rates vom 26.06.2013. Referat4 10-7406-30/15. http://fluechtlingsratthr.de/sites/fluechtlingsrat/files/pdf/gesetze_verordnungen/europa/Lietfaden\%20Umsetzung\%20Verfahrensrichtl inie.pdf. Zug egriffen: 24. Oktober 2016.

Calvot, Thomas, Merat, Claire et al. 2014. Hidden victims of the Syrian crisis: disabled, injured and older refugees.http://www.handicap-international.us/hidden_victims_of_syrian_crisis.ZZugegriffen: 13. Februar 2017. Classen, Georg. 2008. Sozialleistungen für MigrantInnen und Flüchtlinge. Handbuch für die Praxis. Karlsruhe: Von-Loeper-Literaturverlag.

Classen, Georg. 2016. Leitfaden zum Asylbewerberleistungsgesetz. http://www.fluechtlingsinfoberlin.de/fr/asylblg/Leitfaden_AsylbLG.pdf.Zugegriffen: 31. August 2016.

Eichenhofer, Eberhard. 2013. Gesundheitsleistungen für Flüchtlinge. Zeitschrift für Ausländerrecht und Ausländerpolitik: ZAR; Staatsangehörigkeit, Zuwanderung, Asyl und Flüchtlinge, Kultur, Einreise und Aufenthalt, Integration, Arbeit und Soziales, Europa 33 (5/6): 169-174.

Europäische Kommission. 2015. Pressemitteilung. Mehr Verantwortung bei der Bewältigung der Flüchtlingskrise: Europäische Kommission bringt Gemeinsames Europäisches Asylsystem auf Kurs und leitet 40 Vertragsverletzungsverfahren ein. Brüssel, 23. September 2015. http://europa.eu/rapid/press-release_IP-155699_de.htm. Zugegriffen:24.Oktober 2016.

Folz, Friedericke. 2014. Stand des Gemeinsamen Europäischen Asylsystems von Asylsuchenden. In Dokumentation der Fachtagung Besondere Schutzbedürftigkeit von Asylsuchenden feststellen. Rechtliche Bedingungen und Praxismodelle für das Aufnahme- und Asylverfahren. Berlin, 6. Februar 2014, Hrsg. zfm, KommMit für Migranten und Flüchtlinge e.V., Deutsches Rotes Kreuz. Berlin.

\footnotetext{
${ }^{40}$ BT-Drs. 18/7831, S. 5, BT-Drs. 18/9009, S. 3.
} 
Frehe, Horst. 2016. Vorschläge des Forums behinderter Juristinnen und Juristen (FbJJ) zum Kabinettsentwurf eines Gesetzes zur Stärkung des Teilhabe und Selbstbestimmung von Menschen mit Behinderungen (Bundesteilhabegesetz-BTHG).

http://www.reharecht.de/fileadmin/user_upload/RehaRecht/Infothek/Verwaltung_Verb\%C3\%A4nde_Organisati onen/2016/Vorschl\%C3\%A4ge_des_FbJJ_zum_KabE-BTHG-neu-ho.pdf.Zugegriffen am 13. Februar 2017

Frerichs, Konrad. 2014a. § 4 AsylbLGLeistungen bei Krankheit, Schwangerschaft und Geburt. In Juris PraxisKommentar SGB XII. Sozialhilfe/mit AsylbLG, Hrsg. Pablo Coseriu, Wolfgang Eicher, Rainer Schlegel, und Thomas Voelzke. Saarbrücken: Juris Saarbrücken.

Frerichs, Konrad. 2014b. § 6 AsylbLG Sonstige Leistungen. In Juris PraxisKommentar SGB XII. Sozialhilfe/mit AsylbLG, Hrsg. Pablo Coseriu, Wolfgang Eicher, Rainer Schlegel, und Thomas Voelzke. Saarbrücken: Juris Saarbrücken.

Frings, Dorothee. 2009. Werzahlt was? Gesetzliche Grundlagen zur Versorgung psychisch kranker Flüchtlinge. In Forum Hilfen für psychisch krankeFlüchtlinge, Hrsg. Landschaftsverband Rheinland, 53-63.

Frings, Dorothee. 2016. Umsetzung bzw. Anwendung der Aufnahmerichtlinie. Beitrag zur Tagung: Veränderung. Hohenheimer Tage zum Migrationsrecht2016.29-31.01.2016 in Stuttgart-Hohenheim. www.akademiers.de/fileadmin/user_upload/download_archive/migration/20160129_frings_Aufnahmerichtlinie. pdf. Zugegriffen: 16. September 2016.

Hainthaler, Claudia. 2015. Die richtlinienkonforme Rechtsfortbildung im dreistufigen System der Richtlinien wirkungen. Zeitschrift für das Juristische Studium(1): 13-22.

Herbst, Antje. 2015. §§ 4, 6 AsylbLG. In Handbuch der Grundsicherung und Sozialhilfe. Stuttgart: Kohlhammer.

Hohm, Karl-Heinz. 2015. § § 4, 6 AsylbLG. In Kommentar zum Sozialgesetzbuch XII. [SGB XII - Sozialhilfe], Hrsg. Walter Schellhorn, Helmut Schellhorn, Karl-HeinzHohm, Peter Scheider, und Christoph Legros. Köln: Luchterhand.

Kaltenborn, Markus. 2015. Die Neufassung des Asylbewerberleistungsgesetzes und das Recht aufGesundheit. Neue Zeitschrift für Sozialrecht:Zweiwochenschrift für die anwaltliche, betriebliche, behördliche und gerichtliche Praxis 24 (5): 161-165.

Kreienbrock, Lothar und Siegfried Schach. 2012. Epidemiologische Methoden. München u. Heidelberg: Elsevier, Spektrum, Akad. Verlag.

Lachwitz, Klaus. 2013. Art. 25 Gesundheit. In Die UN-Behindertenrechtskonvention in der Praxis. Erläuterungen der Regelung und Anwendungsgebiete, Hrsg. Peter Trenk-Hinterberger und Klaus Lachwitz, 254 280. Köln: Luchterhand.

Lelgemann, Monika und Zahra Mohammadzadeh. 2015. "Flüchtlinge im Regelsystem versorgen". Monika Lelgemann und Zahra Mohammadzadeh vom Bremer Gesundheitsamt zur Gesundheitsversorgung von Flüchtlingen. Soziale Sicherheit: Zeitschrift für Arbeit und Soziales 64 (11):400-404.

Mahler, Claudia. 2014. StellungnahmeDeutsches Institut für Menschenrechte zum Referentenentwurfdes Bundesministeriums für Arbeit und Soziales. Entwurf eines Gesetzes zur Änderung des

Asylbewerberleistungsgesetzes und des Sozialgerichtsgesetzes. www.akademie-

rs.de/fileadmin/user_upload/download_archive/migration/20160129_frings_Aufnahmerichtlinie.pdf.

Zugegriffen:22. September 2016.

Mirza, Mansha, Rene Luna, Bhuttu Mathews, Rooshey Hasnain, Elizabeth Hebert, Allison Niebauer, und Uma Devi Mishra. 2014. Barriers to healthcare access among refugees with disabilities and chronic health conditions resettled in the US Midwest. Journal of immigrant and minority heal th 16 (4): 733-742. doi: 10.1007/s10903013-9906-5.

Mohammadzadeh, Zahra. 1993. Die gesundheitliche Lageund Versorgung der Flüchtlinge in Bremen. Frankfurt a.M.: IKO - Verlag für Interkulturelle Kommunikation.

Mohammadzadeh, Zahra, Felicitas Jung, und MonikaLelgemann. 2016. Health for refugees - the Bremen model. Bundesgesundheitsblatt, Gesund heitsforschung, Gesundheitsschutz 59 (5): 561-569. doi: 10.1007/s00103-016-2331-x.

Müller-Krah, Eva-Maria. 2012. Die Gesundheitsleistungen für Asylbewerbernach §§ 4, 6 AsylbLG gesundheitliches Existenzminimum unterhalb des Existenzminimums? Gesundheit und Pflege: Rechtszeitschrift für das gesamte Gesundheitswesen; 2 (4): 132-143.

Nietha mmer, Andrea und Gerlinde Aumann. 2014. Konzeptionelle Ansätze zur Feststellung besonderer Schutzbedürftigkeit. In Dokumentation der Fachtagung Besondere Schutzbedürftigkeit von Asylsuchenden feststellen. Rechtliche Bedingungen und Praxismodelle für das Aufnahme-und Asylverfahren. Berlin, 6. Februar 2014, Hrsg. zfm, KommMit für Migranten und Flüchtlingee.V., Deutsches Rotes Kreuz, 18-20. Berlin. 
Razum, Oliver, Anne Bunte, Andreas Gilsdorf, Thomas Ziese, und Kayvan Bozorgmehr. 2016a. Zu gesicherten Daten kommen. Deutsches Ärzteblatt 113(4): A 130-A 133.

Razum, Oliver, Anke-Christine Saß, und Kayvan Bozorgmehr. 2016b. Health care for refugees and asylum seekers: challenges and solutions. Bundesgesundheitsblatt, Gesundheitsforschung, Gesundheitsschutz 59 (5): 543-544. doi: 10.1007/s00103-016-2344-5.

Rixen, Stephan. 2015. Zwischen Hilfe, Abschreckung und Pragmatismus: Gesundheitsrecht der Flüchtlingskrise. $\mathrm{Zu}$ den Änderungen durch das Asylverfahrensbeschleunigungsgesetz vom 20.10.2015. Neue Zeitschrift für Verwaltungsrecht: vereinigt mit Verwaltungsrechtsprechung 34 (23): 1640-1644.

Schütte, Wolfgang. 2016. Rechtsgutachten zumReferentenentwurf eines Gesetzes zur Stärkung der Teilhabe und Selbstbestimmung von Menschen mit Behinderung (Bundesteilhabegesetz - BTHG). Erstellt für den Arbeiter-Samariter-Bund Deutschland e.V.(ASB). https://www.asb.de/index.php/download_file/view/2810/1623.Zugegriffen: 24. Oktober 2016.

Schwalgin, Susanne. 2015. Flüchtlingemit Behinderung und ihr Zugang zum deutschen Hilfesystem. Köln: InfoDienste Bundeszentrale für gesundheitliche Aufklärung. http://www.infodienst.bzga.de/?uid=64cc427f39833454db5facf3 dbbd10c5\&id=teaserext9\&idx=5562. Zugegriffen: 16. September 2016.

Spallek, Jacob, Jana Tempes, Hannah Ricksgers, Louisa Marquardt, Luise Prüfer-Kramer, und Alexander Kramer. 2016. The health situation and health care needs of unaccompanied minor refugees - an approximation based on qualitative and quantitative studies from Bielefeld, Germany. Bundesgesundheitsblatt, Gesundheitsforschung, Gesundheitsschutz 59 (5): 636-641. doi: 10.1007/s00103-016-2339-2.

Turhan, Hülya. 2016. Gesundheitsversorgung von geflüchteten Menschen mit Behinderung. Rechtsdienst der Lebenshilfe (3): 151-154.

UN-Fachausschuss für die Rechte von Menschen mit Behinderungen. 2015. Abschließende Bemerkungen überden ersten StaatenberichtDeutschlands. Genf. http://www.institut-fuer-menschenrechte.de/fileadmin/ user_upload/PDF-Dateien/UN-Dokumente/CRPD_Abschliessende_Bemerkungen_ueber_den_ersten Staatenbericht_Deutschlands_ENTWURF.pdf.Zug egriffen: 13. September 2016.

Voigt, Claudius. 2016. Asylbewerberleistungsgesetz: Feindliche Übernahme durch das Ausländerrecht. info also Informationen zum Arbeitslosenrecht und Sozialhilferecht 34 (3): 99-106.

Wächter-Raquet. 2016a. Einführung der Gesundheitskarte für Asylsuchende und Flüchtlinge. Der Umsetzungsstand im Überblick derBundesländer.https://www.bertelsmannstiftung.de/fileadmin/files/BSt/Publikationen/GrauePublikationen/Studie_VV_Gesundheitskarte_Fluechtlinge_2 016.pdf. Zugegriffen: 13. Juli 2016.

Wächter-Raquet. 2016b. Überblick Stand: Mai 2016. Einführung der Gesundheitskarte für Asylsuchende und Flüchtlinge. Der Umsetzungsstand im Überblick der Bundesländer. https://www.bertelsmannstiftung.de/fileadmin/files/Projekte/Integrierte_Krankenversicherung/Ueberblick_Gesundheitskarte_Fluechtlinge Mai2016.pdf.Zugegriffen: 13. Juli 2016.

Wahrendorf, Volker. 2014. § 4 AsylbLG. In SGB XII. Sozialhilfe mit Asylbewerberleistungsgesetz; Kommentar, Hrsg. Christian Grube, Volker Wahrendorf, Karin Bieback, Thomas Flint, und Klaus Streichsbier. München: Beck. 